\title{
Cecum location in rats and the implications for intraperitoneal injections
}

\author{
Genaro A. Coria-Avila, DVM, MSc, $P h D^{1,2}$, Alex M. Gavrila, $B A^{1}$, Shann Ménard, $B A^{1}$,
} Nafissa Ismail, $M A^{1}$ \& James G. Pfaus, $P h D^{1}$

Intraperitoneal injection is a common route for parenteral administration of drugs in rodents. A serious consequence associated with this technique, however, is the puncture of vital organs such as the cecum, which causes pain and occasionally peritonitis. Reports have described the cecum as located on either side of the lower abdominal cavity, contributing to the idea that intraperitoneal injections can be performed in either side. The authors investigated the location of the cecum in adult male and female albino and pigmented rat strains, and evaluated the consequences of intraperitoneal injections in the right and left portion of the lower abdomen. Of the rats they investigated, $71.8 \%$ had ceca on the left side of the abdomen. The authors also found that injections on the left side were more likely to result in punctured ceca.

Intraperitoneal (i.p.) injection is a common route for parenteral administration of drugs in laboratory rodents. The i.p. technique allows for the deposition of substances into the peritoneal cavity, which is formed by the peritoneum, a serous membrane that covers the internal walls of the abdominal cavity (parietal peritoneum) and most of the abdominal organs (visceral peritoneum). Under normal circumstances the peritoneum is transparent and smooth, functioning to prevent friction between the moving viscera, such as the intestines. The peritoneum also maintains the abdominal organs in place and acts as a medium for their blood and lymphatic vessels.

In rats, substances deposited into the peritoneal cavity are absorbed by vessels such as the colic, the intestinal, and the mesenteric veins, which converge into the anterior mesenteric vein that carries blood via the portal system into the liver ${ }^{1}$. After being metabolized in the liver, i.p.-injected substances are carried with deoxygenated blood by the inferior cava vein into the right atrium of the heart. The pulmonary artery distributes this blood to the lungs to be oxygenated, returning via the pulmonary vein into the left atrium and then to the left ventricle and finally into the circulatory system at large. Despite this seemingly lengthy process, the speed of absorption following i.p. injections is between $25 \%$ and $50 \%$ the speed of intravenous (i.v.) injection ${ }^{2}$. For this reason, i.p. injections are preferred over i.v. injections in rats when the breakdown of drugs in the liver is not an issue for the research.

The i.p. technique is simple and does not require much training. Rats can be injected daily over the course of several days (for 3-4 weeks, for example), and with the proper precautions this daily procedure does not cause any serious complications. However, even a single poorly administered i.p. injection can have adverse consequences, including lesions of the internal organs and peritonitis (inflammation of the peritoneum). This is not only painful for the rats, but can lead to death if bacteria from the intestines enter the circulatory system, causing bacteremia and septic shock.

Given the potential for complications from i.p. injections, all personnel who perform this procedure in laboratory rats should be trained by a veterinarian or an experienced laboratory animal care technician. The training procedure should include an appropriate explanation of the technique, as well as a review of the abdominal anatomy, so that trainees understand the correct location of organs that can potentially be punctured by the needle (Fig. 1). The cecum, for example,

${ }^{1}$ Center for Studies in Behavioral Neurobiology, Department of Psychology, Concordia University, 7141 Sherbrooke West, Montréal, QC H4B 1R6, Canada. ${ }^{2}$ Instituto de Neuroetología, Universidad Veracruzana, Av. Dr. Luis Castelazo s/n, Col. Industrial Las Animas, Xalapa, Veracruz C.P.91190, Mexico. Correspondence should be addressed to G.A.C.-A. (coria75@yahoo.com). 


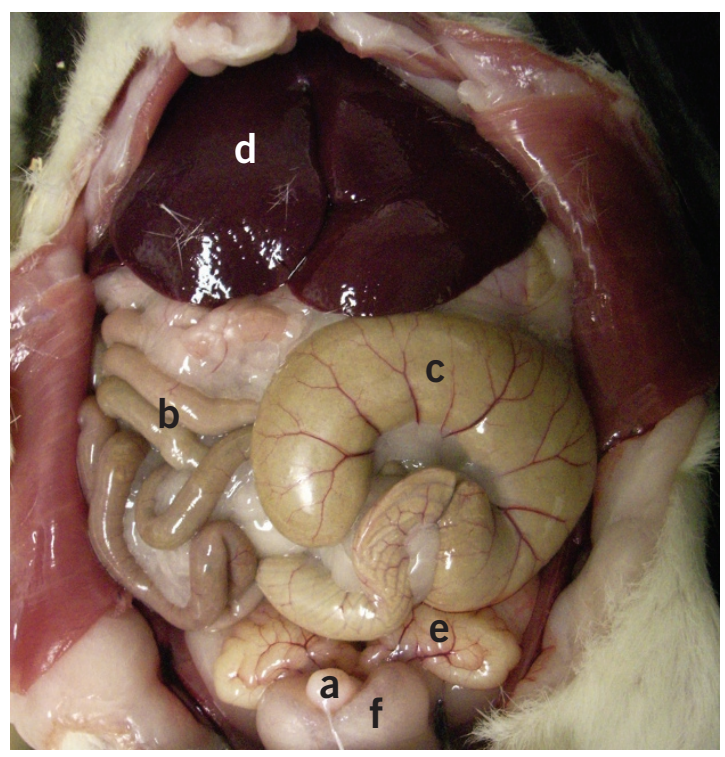

FIGURE 1 | The (a) bladder, (b) small intestines, (c) cecum, and (d) liver are among the organs that can be punctured during i.p. injections. Males can also be punctured in the (e) seminal glands and (f) prostate. In the case of females, although unlikely, punctures can occur in the uterine horns.

is the largest structure in the lower abdominal cavity, and the distension of its walls make it more likely to be pierced by a needle relative to the less distended walls of the small intestine. Unfortunately, there are no reliable sources of information with regard to the cecum location and the appropriate side for injections.

Recently, we reviewed the protocol for i.p. injections reported in two textbooks on the laboratory rat ${ }^{3,4}$. Given the popularity of these books, it is possible that they are among the main reference sources for rat surgical procedures and handling techniques among many laboratory animal professionals. As mentioned above, the i.p. injection technique is simple and easy to learn. However, we feel that the explanations provided in these two textbooks are not sufficient and may lead to confusion and errors when readers are trying to learn and apply the procedures.

Nebendahl ${ }^{4}$ indicates that the abdomen can be divided into four quadrants by creating one imaginary midline and one perpendicular line at the level of the umbilical mark, and that i.p. injections should be given into the lower-left quadrant in order to avoid an accidental perforation of vital organs such as the cecum. Although this explanation does not identify whether the left or right quadrant is in reference to the rat or in relation to the researcher, Nebendahl's chapter includes a picture that illustrates a person performing the i.p. injection in the lower-left quadrant of the rat's abdomen. This implies that the organs that can be potentially punctured (such as the cecum) are located in the lower-right quadrant of the rat, but this conclusion is not supported by an anatomical study, although it refers to the textbook by Waynforth and Flecknell ${ }^{3}$.

Waynforth and Flecknell ${ }^{3}$ explain that injections have to be given into the lower-left quadrant of the abdomen. However, the picture that they show illustrates a person performing an i.p. injection into the lowerright quadrant of the rat. Thus, for these authors, it would appear that 'lower' and 'left' refer to the quadrant observed from the handler's perspective and not from the rat's perspective. These two references suggest the lower-left quadrant as the correct place to inject, but apparently differ as to the definition of 'lower-left quadrant'. Given this inconsistency, it is not surprising to find that $11-20 \%$ of the i.p. injections performed by skilled researchers may be improperly located ${ }^{5}$. Data from our laboratory indicate that when errors occur, the organ most often affected is the cecum (a digestive organ containing commensal bacteria that break down complex polysaccharides, such as cellulose, into simpler monosaccharides ${ }^{6}$; Fig. 2). Other reported errors include perforation of the stomach, intestines, subcutaneous or intramuscular areas, retroperitoneum, and bladder ${ }^{7}$.

Given that the rat cecum is homologous to the large cecum in other species (like horses) or to the appendix in humans, one might expect to find it in the lowerright quadrant of the rat's abdomen. Thus, we asked whether the lower-left quadrant of the rat is the more appropriate location for i.p. injections. To answer this question, we carried out a practical study to describe the correct location of the cecum and the likelihood of it being punctured during i.p. injections in adult male and female Wistar and Long Evans rats.

\section{METHODS}

\section{Animals}

We used 289 adult rats (Long Evans females $(N=46)$; Long Evans males $(N=82)$; Wistar females $(N=95)$; Wistar males $(N=66))$. Both males and females were about to be euthanized after being used in studies of sexual behavior. The males were intact and the females were ovariectomized. Of these rats, only the females had previously received i.p. injections (at least four months earlier in preparation for ovariectomy). Thus, the probability of observing previous lesions due to i.p. injections in the abdominal cavity was very small for the females and nonexistent for the males. All animal procedures conformed to the guidelines of the Canadian Council on Animal Care, and were approved by the Concordia University Animal Research Ethics Committee.

\section{Injection protocol}

We randomly assigned males and females of the two strains to three groups: one group received i.p. injections 
a

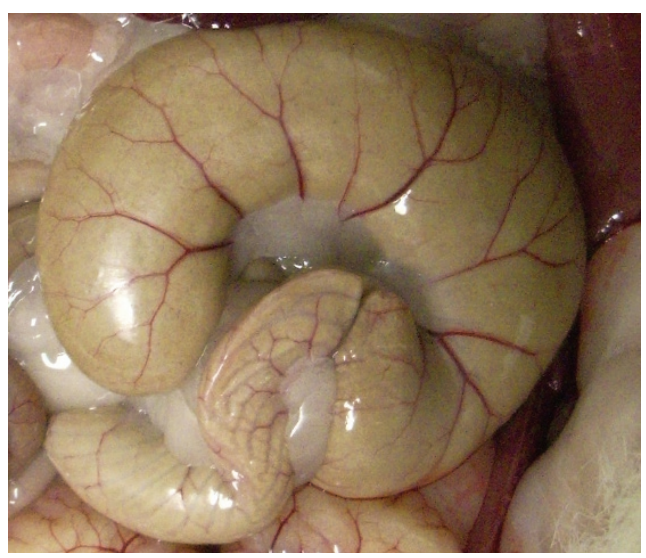

b

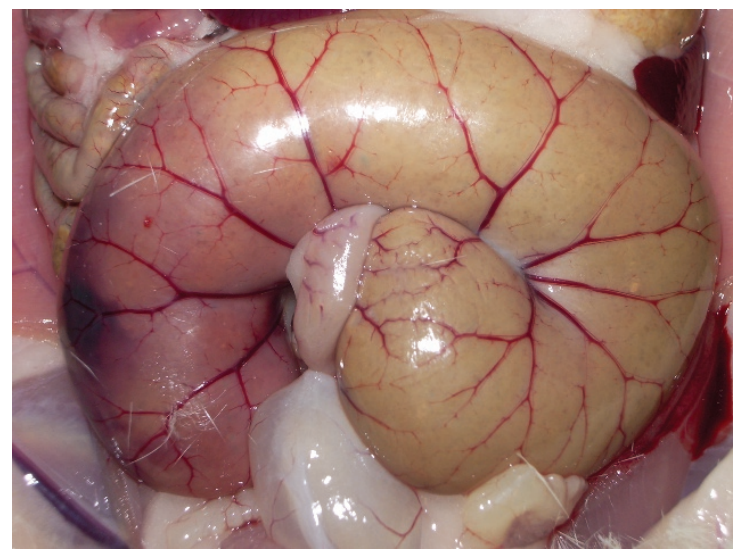

C

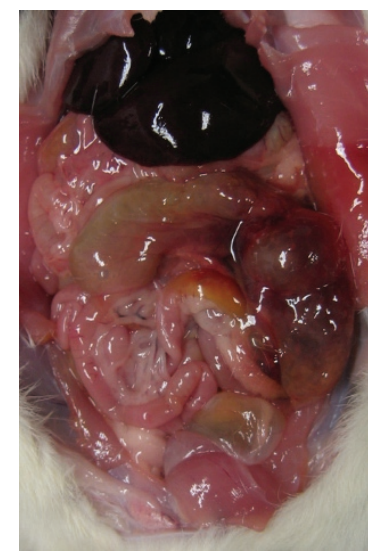

FIGURE 2 |The cecum is a blind sac (a) that is located between the end of the ileum and beginning of the large intestine. (b) When the cecum is punctured superficially with a needle, inflammation may occur, causing pain. (c) When the puncture trespasses all the way through the cecum, small quantities of its fecal contents (including microorganisms) can leak out into the peritoneum. When this occurs, peritonitis is likely to occur within the following 24-96 h.

of $1 \mathrm{ml}$ of saline $(0.9 \%)$ in the lower-right area of the abdominal cavity; a second group received injections in the lower-left area (Fig. 3); the control group did not receive injections. One person, skilled in the i.p. procedure, performed all of the injections. We used a 1-ml syringe with a 26 G, 3/8-inch needle. During the injection, the operator restrained the rat with the nondominant (in this case left) hand by the thorax so that the rat was in a vertical position and the rat's abdomen faced the person performing the injection (Fig. 4).

Following the i.p. injection, we left the animal in its home cage for $5 \mathrm{~min}$ so that potential lesions would be observed as hemorrhages or swelling. We then euthanized the rats with $\mathrm{CO}_{2}$ and immediately performed gross necropsies to detect the locations of the ceca and describe any potential lesions caused by the i.p. injections. In most cases, the cecum was clearly located either in the right or left of the cavity. However, when the location was not obvious, we categorized the cecum as on the right or left side of the abdominal cavity depending on the location of the three-quarters of the cecum proximal to the junction with the intestine. When more than one quarter was located in the other side, we regarded the cecum as in the middle. We recorded the presence of a lesion if we observed any sign of inflammation or hemorrhage around the site of injection.

\section{Statistical analyses}

We subjected the results to a Chi-squared statistical analysis to assess the proportion of animals with the cecum on the right, left, or in the middle. Furthermore, the proportions of animals with lesions were compared between those injected on the right or left side. The level of significance was set at $P<0.05$.

\section{RESULTS}

\section{Cecum location by strain}

Table 1 indicates the number of rats that had ceca located on the left, right, or in the middle of the abdominal cavity. In all four groups there were significant differences, with the cecum most commonly located in the left part of the abdominal cavity. The

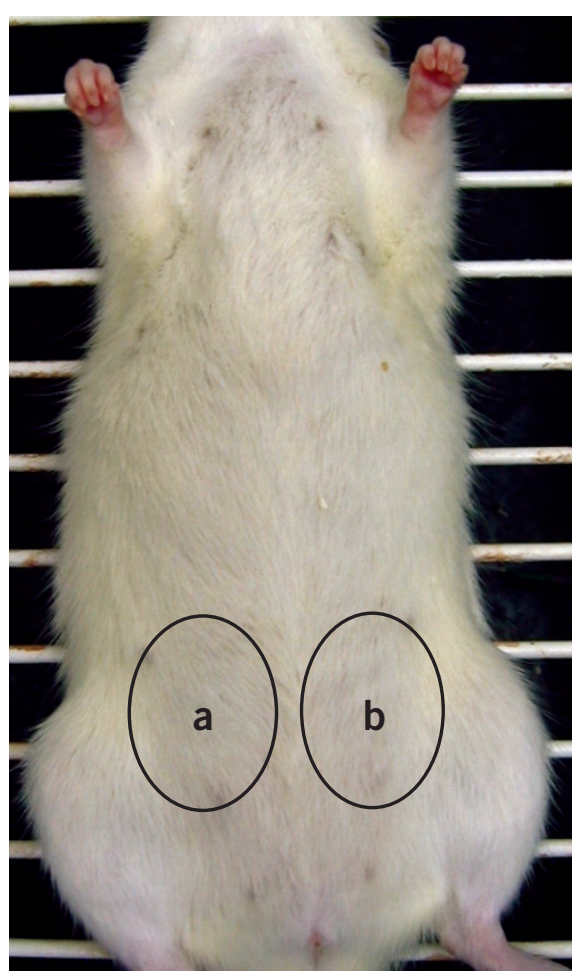

FIGURE 3 | Sites of injections in our research protocol; (a) lower-right side, (b) lower-left side. 


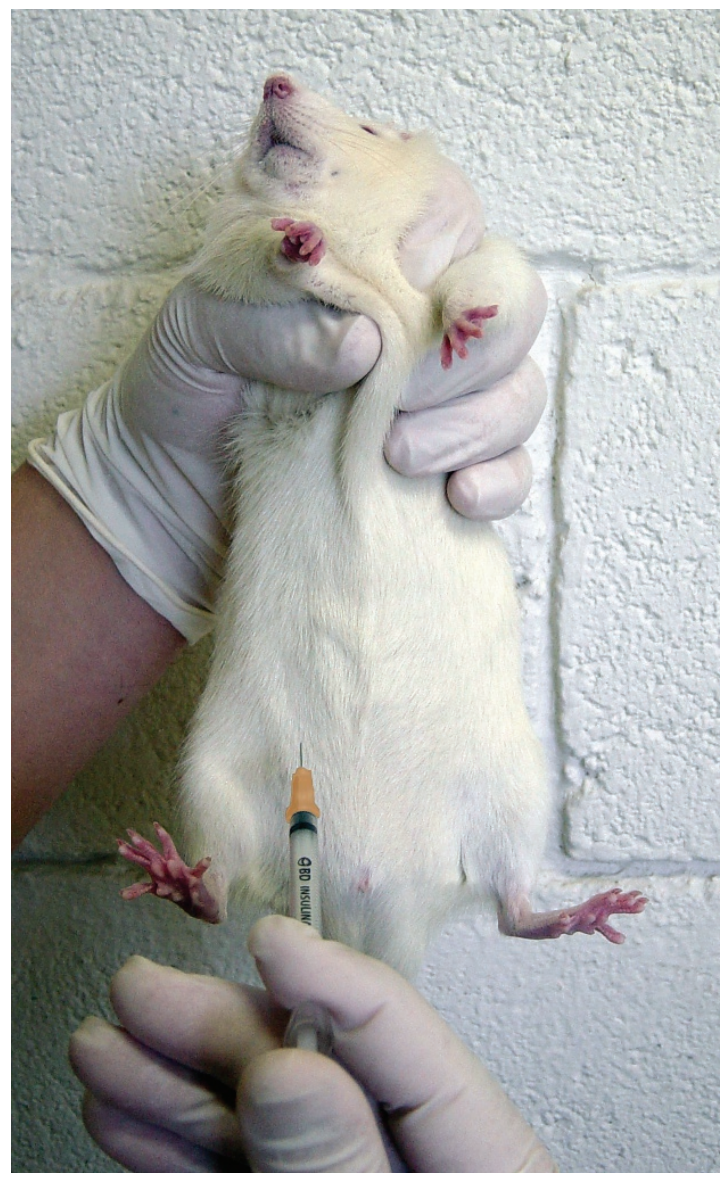

FIGURE 4 | Intraperitoneal injection in the lower-right quadrant of the rat. We used a 1-ml syringe with a $26 \mathrm{G}, 3 / 8$ inch needle. During the injection, the rat was restrained with the nondominant (in this case left) hand by the thorax, so that it was in a vertical position with the abdomen toward the person performing the injection.

results indicate that $60.9 \%$ of Long Evans females had ceca located on the left, compared to $21.7 \%$ on the right, and $17.4 \%$ in the middle. A Chi-squared analysis revealed significant differences in these proportions $\left(\chi^{2}(2, N=46)=15.8, P<0.001\right)$. In the case of Long Evans males, the results indicate that $51.2 \%$ had ceca located on the left, compared to $30.5 \%$ on the right, and $18.3 \%$ in the middle. The Chi-squared analysis revealed significant differences in these proportions $\left(\chi^{2}(2, N=82)=13.63, P<0.001\right)$. For the Wistar females, $73.7 \%$ had ceca located on the left, compared to $15.8 \%$ on the right, and $10.5 \%$ in the middle. The analysis revealed significant differences in these proportions $\left(\chi^{2}(2, N=95)=70, P<0.001\right)$. For the Wistar males, $59.1 \%$ had ceca located on the left, compared to $30.3 \%$ on the right, and $10.6 \%$ in the middle. The analysis revealed significant differences in these proportions $\left(\chi^{2}(2, N=66)=23.5\right.$, $P<0.001)$.
Given that the probability for the cecum to be located in the middle was small and that such a small probability may have caused the significant differences in proportion between the three locations in the previous analysis, we decided to run another comparison exclusively for the probability of the cecum to be located either on the right or left. This second comparison allowed us to observe real differences in cecum location between right and left quadrants. The results indicate that $73.6 \%$ of Long Evans females had ceca on the left and $26.3 \%$ on the right. Chi-squared analysis revealed a significant difference in this proportion $\left(\chi^{2}(1, N=38)=8.5, P=0.004\right)$. In the case of Long Evans males there were also significant differences; $62.6 \%$ of them had ceca located on the left and $37.3 \%$ on the right $\left(\chi^{2}(1, N=67)=4.3, P=0.03\right)$. In the case of Wistar females, $82.3 \%$ had ceca on the left and only $17.6 \%$ on the right $\left(\chi^{2}(1, N=85)=35.5, P<0.001\right)$. In the case of Wistar males, $66.1 \%$ had ceca on the left, and $33.8 \%$ on the right $\left(\chi^{2}(1, N=59)=6.11, P=0.01\right)$.

\section{Cecum location by gender}

We also ran a comparison using only the Wistar and Long Evans males and another comparison exclusively for the females. The results indicate that $64.2 \%$ of the males had ceca located on the left side and $35.7 \%$ on the right. The statistical analysis revealed significant differences in these proportions $\left(\chi^{2}(1, N=126)=10.2, P<\right.$ $0.001)$. With regard to females, $79.7 \%$ had ceca located on the left and $20.3 \%$ on the right. The statistical analysis also revealed that these differences were significant $\left(\chi^{2}(1, N=123)=43.3, P<0.001\right)$.

\section{Cecum location overall}

Finally, we decided to run a comparison of all the rats in the study. The results indicate that $71.8 \%$ of the rats had ceca located on the left and $28.1 \%$ on the right (Fig. $5)$. The Chi-squared analysis indicates that these differences were statistically significant $\left(\chi^{2}(1, N=289)\right.$ $=47, P<0.001)$.

\section{Lesions caused by intraperitoneal injections}

With regard to lesions caused by i.p. injections, the results indicate that of the total 127 injections, 8 $(6.29 \%)$ caused a lesion. Of the 64 rats injected on the left side of the abdomen, 4 had an observable lesion in the cecum (6.25\%) located on the left side (Fig. 5). Of the total of 63 rats injected on the right, 4 had an observable lesion, only one of which lesion (1.58\%) was in the cecum. In that case, the cecum was located in the middle of the abdominal cavity. The other lesions were reported as swelling of the abdominal wall or small hemorrhages. There were no observable lesions in other organs of the cavity. In addition, there were no signs of preexisting lesions caused by i.p. injections in the females. 


\begin{tabular}{|c|c|c|c|c|c|c|c|}
\hline \multicolumn{3}{|c|}{ Cecum } & \multirow[b]{2}{*}{$\%$ (PER GROUP) } & \multicolumn{2}{|c|}{ Injection } & \multicolumn{2}{|l|}{ Lesions } \\
\hline SUBJECTS & LOCATION & $N$ & & LOCATION & $N$ & \% (OF 127 RATS INJECTED) & $N$ \\
\hline \multirow[t]{9}{*}{ Long Evans (female) } & \multirow[t]{3}{*}{ Left } & \multirow[t]{3}{*}{28} & \multirow[t]{3}{*}{60.9} & Left & 5 & 0.0 & 0 \\
\hline & & & & Right & 4 & 0.0 & 0 \\
\hline & & & & None & 19 & 0.0 & 0 \\
\hline & \multirow[t]{3}{*}{ Right } & \multirow[t]{3}{*}{10} & \multirow[t]{3}{*}{21.7} & Left & 0 & 0.0 & 0 \\
\hline & & & & Right & 0 & 0.0 & 0 \\
\hline & & & & None & 10 & 0.0 & 0 \\
\hline & \multirow[t]{3}{*}{ Middle } & \multirow[t]{3}{*}{8} & \multirow[t]{3}{*}{17.4} & Left & 0 & 0.0 & 0 \\
\hline & & & & Right & 0 & 0.0 & 0 \\
\hline & & & & None & 8 & 0.0 & 0 \\
\hline \multirow[t]{9}{*}{ Long Evans (male) } & \multirow[t]{3}{*}{ Left } & \multirow[t]{3}{*}{42} & \multirow[t]{3}{*}{51.2} & Left & 9 & 1.6 & 2 \\
\hline & & & & Right & 6 & 0.0 & 0 \\
\hline & & & & None & 27 & 0.0 & 0 \\
\hline & \multirow[t]{3}{*}{ Right } & \multirow[t]{3}{*}{25} & \multirow[t]{3}{*}{30.5} & Left & 5 & 0.0 & 0 \\
\hline & & & & Right & 6 & 0.0 & 0 \\
\hline & & & & None & 14 & 0.0 & 0 \\
\hline & \multirow[t]{3}{*}{ Middle } & \multirow[t]{3}{*}{15} & \multirow[t]{3}{*}{18.3} & Left & 2 & 0.0 & 0 \\
\hline & & & & Right & 4 & 0.8 & 1 \\
\hline & & & & None & 9 & 0.0 & 0 \\
\hline \multirow[t]{9}{*}{ Wistar (female) } & \multirow[t]{3}{*}{ Left } & 70 & 73.7 & Left & 9 & 0.8 & 1 \\
\hline & & & & Right & 13 & 1.6 & 2 \\
\hline & & & & None & 48 & 0.0 & 0 \\
\hline & Right & 15 & 15.8 & Left & 2 & 0.0 & 0 \\
\hline & & & & Right & 2 & 0.0 & 0 \\
\hline & & & & None & 11 & 0.0 & 0 \\
\hline & Middle & 10 & 10.5 & Left & 4 & 0.0 & 0 \\
\hline & & & & Right & 0 & 0.0 & 0 \\
\hline & & & & None & 6 & 0.0 & 0 \\
\hline Wistar (male) & Left & 39 & 59.1 & Left & 18 & 0.8 & 1 \\
\hline & & & & Right & 17 & 0.0 & 0 \\
\hline & & & & None & 4 & 0.0 & 0 \\
\hline & Right & 20 & 30.3 & Left & 7 & 0.0 & 0 \\
\hline & & & & Right & 8 & 0.8 & 1 \\
\hline & & & & None & 5 & 0.0 & 0 \\
\hline & Middle & 7 & 10.6 & Left & 3 & 0.0 & 0 \\
\hline & & & & Right & 3 & 0.0 & 0 \\
\hline & & & & None & 1 & 0.0 & 0 \\
\hline & Total & 289 & & & 289 & 6.30 & 8 \\
\hline
\end{tabular}

\section{DISCUSSION}

Our results indicate that a greater proportion of rats have ceca located on the left side $(71.8 \%)$ of their lower abdominal cavity relative to the right side (28.2\%). Given that the cecum is the largest organ within this area, the probability of suffering a puncture is higher when i.p. injections are given on the left side of the abdominal wall. Consequently, we suggest that the appropriate side for i.p. injections is in the lower-right quadrant of the rats' abdomen. It is interesting to note that random injections in either side of the abdomen did not result in a different percentage of animals with lesions. However, explicit lesions of the cecum were more likely to occur with injections performed on the left side, whereas other types of lesions (such as abdominal hemorrhage) were found with the injections performed on the right side.

The reason some animals have ceca located on the left side and others on the right is not known. Our observations indicate that there are no ligaments that keep the cecum fixed, with the exception of the iliocecal ligament that keeps the apex attached to the ileum. When the cecum is located on the left side of the abdomen, its curvature commonly projects laterally, then rostrally, and then medially; thus the cecum's apex is always close to the midline of the abdomen, as in Figure 1. When the cecum is located on the right side of the abdomen, it appears to maintain the same curvature but is shifted 


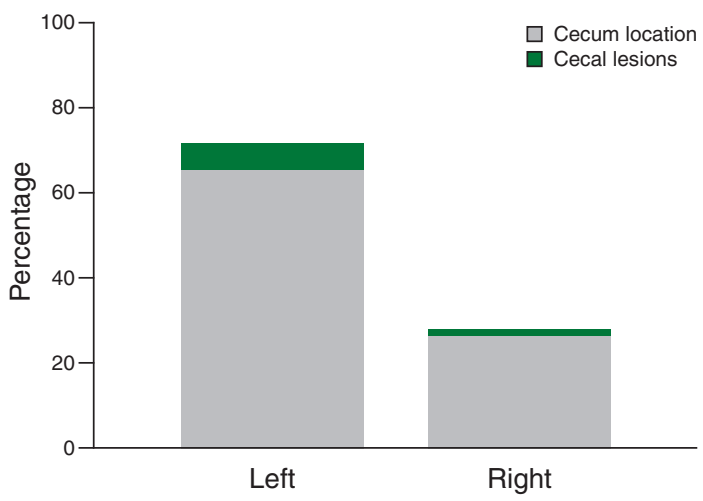

FIGURE 5 | Cecum location and lesions caused by i.p. injections in rats. Most of the rats have ceca located in the lower-left side of the abdominal cavity. Injections performed on the lower-left side resulted in cecum punctures about $6.25 \%$ of the time. In contrast, injections in the lower-right abdomen resulted in punctures to this organ only $1.58 \%$ of the time.

to the right, so that the apex is no longer pointing at the midline, but rather to the right abdominal wall toward the kidney. However, occasionally, when the cecum is on the right side, the apex curvature can project laterally, then caudally, and then medially.

Based on early observations of our study, we hypothesized that cecum location may depend on the amount of fat within the cavity. Thus, in an obese rat, the cecum is more likely to be on the right side because the accumulation of intra-abdominal fat physically pushes the organ to that location. However, we do not have empirical evidence to confirm this conjecture. Furthermore, we emphasize that our research protocol did not allow us to rule out the possibility that the cecum location was the result of postmortem shifting. Antemortem microcomputed tomography (micro-CT) or magnetic resonance imaging (MRI) could possibly be used to confirm our findings.

Given that $62-72 \%$ of the rats had ceca located on the left side of the abdomen, we advise giving injections in the lower-right quadrant of the rat's abdomen. However, other safety measures can be added to the i.p. protocol. These include the use of the adequate needle size ( $26 \mathrm{G}, 3 / 8$-inch, for example), and the use of stoppers. The latter can be easily made out of the needle cap, which can be cut transversally so that it can still be screwed to the bottom of the needle. The tip of the needle should project about $4-5 \mathrm{~mm}$ beyond the border of the stopper, which prevents insertion of the total length of the needle into the cavity. Shorter needles may result in intramuscular injections of the abdominal walls.

Tilting the rat so that its head is lower than the rest of its body has been recommended during i.p. injections; however, in agreement with $\mathrm{Nebendhal}^{4}$, we believe that this position is not always feasible. Many researchers or students work alone and with rats that are large (350-500 g). Older rats can reach a bodyweight of 500$700 \mathrm{~g}$ and may be difficult to handle in that position, especially by the back skin. We therefore recommend holding the rat as we did in the present study, restrained with the nondominant hand by the thorax, so that the rat is in a vertical position with the abdomen toward the person performing the injection (Fig. 4).

\section{CONCLUSIONS}

We suggest that the most appropriate site for i.p. injections in rats is the lower-right quadrant of the rat's abdomen. According to our data, this procedure results in lesions to the abdominal walls in only $4.76 \%$ of injections and lesions to the cecum in only $1.58 \%$ of injections.

\section{COMPETING INTERESTS STATEMENT}

The authors declare no competing financial interests.

Received 2 January 2007; accepted 23 March 2007. Published online at http://www.labanimal.com

1. Wells, T.A.G. The Rat: A Practical Guide (Heinemann Educational Books, London, UK, 1964).

2. Woodard, G. in Methods of Animal Experimentation (ed. Gay, W.J.) 343-359 (Academic Press, New York, 1965).

3. Waynforth, H.B. \& Flecknell, P.A. Experimental and Surgical Techniques in the Rat (Academic Press, London, 1992).

4. Nebendahl, K. in The Laboratory Rat (ed. Krinke, G.J.) 463-484 (Academic Press, London, UK, 2000).

5. Lewis, R.E., Kunz, A.L. \& Bell, R.E. Error of intraperitoneal injections in rats. Lab. Anim. Care 16(6), 505-509 (1966).

6. Yang, M.G., Manoharan, K. \& Young, A.K. Influence and degradation of dietary cellulose in cecum of rats. J. Nutr. 97(2), 260-264 (1969).

7. Claassen, V. Neglected Factors in Pharmacology and Neuroscience Research (Elsevier, Amsterdam, 1994). 